MATEC Web of Conferences 22,03002 (2015)

DOI: $10.1051 /$ matec conf/ 20152203002

(C) Owned by the authors, published by EDP Sciences, 2015

\title{
Design and Experimental Research of the Anti-sway Test Bed for Off- shore Crane
}

\author{
Shenghai Wang*, Haiquan Chen, Xinyang Chen \& Yan Shen \\ College of Marine Engineering, Dalian Maritime University, Dalian, Liaoning, China
}

\begin{abstract}
Offshore cranes are commonly used in cargo handling, offshore installation and replenishment at sea. However, because of the wave motion of the ship and the flexibility of the rope, the sway of payload is unavoidable and may put relevant operations in danger. A sling tray mechanical anti-sway system is proposed in this paper aiming to reduce the payload sway of some buoy tender. An anti-sway test bed is designed, analyzed and built to testify the proposed anti-sway system and lay the foundation of future practical application.
\end{abstract}

Keywords: design; test bed; anti-sway; offshore crane

\section{INTRODUCTION}

Offshore cranes are commonly used in cargo handling, offshore installation and replenishment at sea. However, because of the wave motion of the ship and the flexibility of the rope, the sway of payload is unavoidable and may put relevant operations in danger.

Two main methods at present exist to restrain payload sway of offshore crane: one is to drag the middle of the rope with tagline, which is often achieved by installing mechanical anti-sway system on offshore crane; the other is to design anti-sway controller which controls the crane tip motion to compensate the ship motion and restrain the payload sway based on the motion measurement of ship, crane and payload ${ }^{[1]}$, and it needs no extra mechanical structure except the slew, luff, hoist mechanism of the crane.

One commonly used mechanical anti-sway system is Maryland Rigging, Kimiaghalam B built its linear dynamical model based on Lagrange method, and restrained the payload sway effectively by applying model predictive control ${ }^{[2]}$ and optimum control ${ }^{[3]}$ Another mechanical anti-sway system is Rider Block Tagline System (RBTS) which is much simpler than Maryland Rigging in both control and structure, but it will be limited when the rope is too long. Parker designed an active rider block tagline system (ARBTS) ${ }^{[4]}$ and compared it with Pendulation Control System ${ }^{[5]}$, it was found that using ARBTS control strategy the payload could be maintained in a fixed inertial position using $80 \%$ less power compared to using the slew, luff, and hoist strategy alone.

In terms of anti-sway controller design, Masoud ${ }^{[6]}$ designed a controller based on delayed feedback of the angles of the cargo-hoist cable in and out of the plane of the boom and crane tower, the payload sway was significantly reduced by controlling the slew and luff angles of the boom. Jang ${ }^{[7]}$ built dynamic model of ship-mounted container crane, and designed a T-S fuzzy controller to reduce the payload sway. Ismail ${ }^{[8]}$

*Corresponding author: wsh2011712@sina.com built the three-dimensional model of offshore boom crane, designed a second order sliding mode controller which could track to expect trajectory while reducing the payload sway. In addition, the mechanical shock was reduced as well. Fang ${ }^{[9]}$ designed a nonlinear controller based on the dynamic model of offshore boom crane built by Wang ${ }^{[1]}$, and applied Lyapunov method to prove its stability, and finally carried out experiment to testify its performance.

A sling tray mechanical anti-sway system (STMAS) is proposed in this paper aiming to reduce the payload sway of some buoy tender. An anti-sway test bed is designed, analyzed and built to testify the proposed anti-sway system and lay the foundation of future practical application.

\section{DESIGN OF ANTI-SWAY TEST BED}

\subsection{Structure of STMAS}

On the basis of RBTS, a sling tray mechanical anti-sway system (STMAS) is proposed in this paper. Its structure is shown as Figure.1. The STMAS contains a sling tray, two taglines, two guide arms, three suspension lines of sling tray and a hoist line of sling tray. The hoist line of sling tray is driven by a motor to lift or lower the sling stray. The two taglines are respectively guided by a guide arm and driven by a motor to keep the necessary tension. The hoist line of payload passes through the hole in the middle of the sling tray. The principle of STMAS is actually quite simple: the sling tray can block payload pendulum motion around the crane tip, thus shortening the radius of the pendulum motion and reducing the payload sway. The hole of the sling tray allows certain slightly sway of the rope, and the taglines allow certain slightly sway of the sling tray, thus to prevent the taglines from too much shock. 


\section{MATEC Web of Conferences}

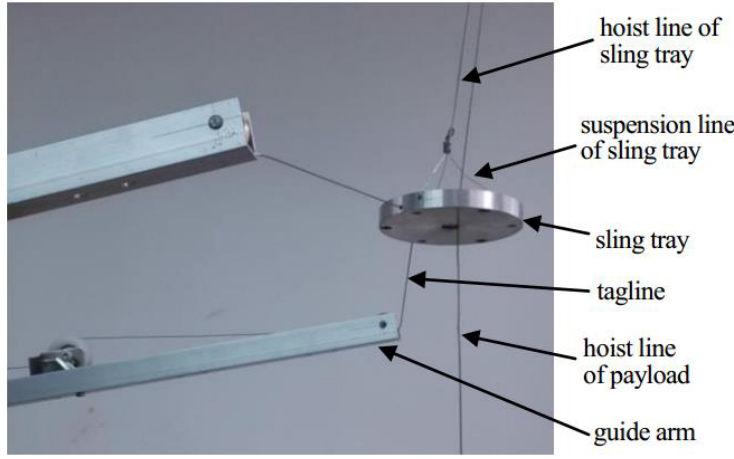

Figure 1. Structure of STMAS

In order to testify the performance of the proposed STMAS, an anti-sway test bed for offshore crane is designed and built based on an existed ship motion simulator, and the process can be divided into four steps:

(1) Mechanical design of the scale crane with the STMAS;

(2) Electrical design of the scale crane with the STMAS;

(3) Design of sway angle measurement device;

(4) Assemble the components to build an anti-sway test bed for offshore crane.

These steps will be introduced in detail in the following passage.

\subsection{Mechanical design of the scale crane with the STMAS}

Since a ship motion simulator has already built in Mechatronic Lab in Dalian Maritime University, the main task of mechanical design here is to design a scale crane with a STMAS. Its three dimensional structure is shown as Figure 2.

In Figure 2, the overall structure can be divided into three parts:

(1) General crane operational mechanisms including a slew motor, a reducer, a rotating shaft, a luff motor, a hoist motor and a hoist line of payload;

(2) Structural supports including a base, a supporting platform and a pedestal;

(3) The STMAS which consists of a sling tray, three suspension lines, a hoist line of sling tray, a hoist motor of sling tray, two taglines, two guide arms and two tagline motors.

The guide arms and tagline motors are installed symmetrically on the supporting platform. There are several holes on the bottom of the base which will be used to install the scale crane on the ship motion simulator later. The main function of the tagline motor is to pull or release the tagline while keeping necessary tension in the tagline. The hoist motor of sling tray is used to lift or lower the sling tray. The luff, slew and hoist operations are carried out by the luff motor, slew motor and hoist motor of payload.
In order to keep necessary tension in the tagline, the position of sling tray must be controlled synchronously with payload when carrying out luff or payload hoist operations. This problem will be analyzed in detail in section 3

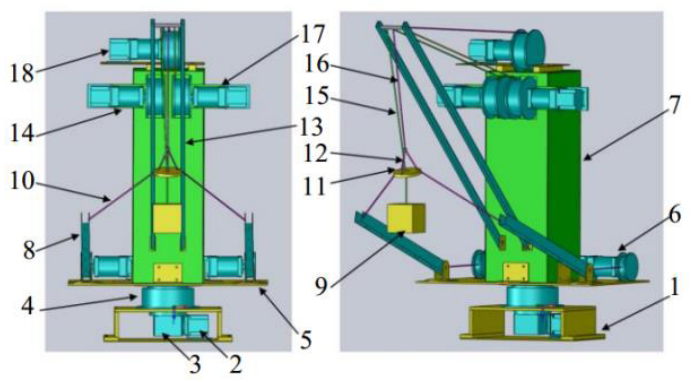

Figure 2. Three-dimensional structure of the scale crane with the STMAS

1-base, 2-slew motor, 3-reducer, 4-rotating shaft, 5-supporting platform, 6-tagline motor, 7-pedestal, 8-guide arm, 9-payload, 10-tagline, 11-sling tray, 12-suspension line, 13-boom, 14-luff motor, 15-hosit line of payload, 16-hoist line of sling tray, 17-hoist motor of sling tray, 18-hoist motor of payload.

\subsection{Electrical design of the scale crane with the STMAS}

In Figure 2, it is quite clear that six motors must be driven and controlled, so the basic task of electrical design is to supply power and send control command to these motors, and receive feedback signal from these motors as well. The systematic block diagram of electrical system is shown in Figure 3.

In Figure 3, the product type of the control card is PCI-1285E which is produced by Advantech. The control card is DSP-based SoftMotion PCI bus controller card and features 8-axis design which means it can control 8 motors simultaneously. The terminal board ADAM-3956 is produced by Advantech as well The terminal board features 4-axis design which means two terminal boards are necessary to match one control card for six motors control in Figure 3. The product type of the actuator is HBS507 and the product type of the motor is 573HBM20-1000, both of which are produced by Leadshine Technology.

The control card is inserted in the general PCI of the computer. The control card and the terminal board are connected by PCL-101100SB which is a 100-pin shielded cable. The two terminal boards are powered by $5 \mathrm{~V}$ switching power supply whereas the six actuators are powered by the $36 \mathrm{~V}$ switching power supply. The terminal CCW-/DIR-, CCW+/DIR+, CW-/PULS-, $\mathrm{CW}+$ PULS + of the terminal board is connected to the terminal DIR-, DIR+, PUL-, PUL+ of the actuator respectively. These terminals represent four pulse 


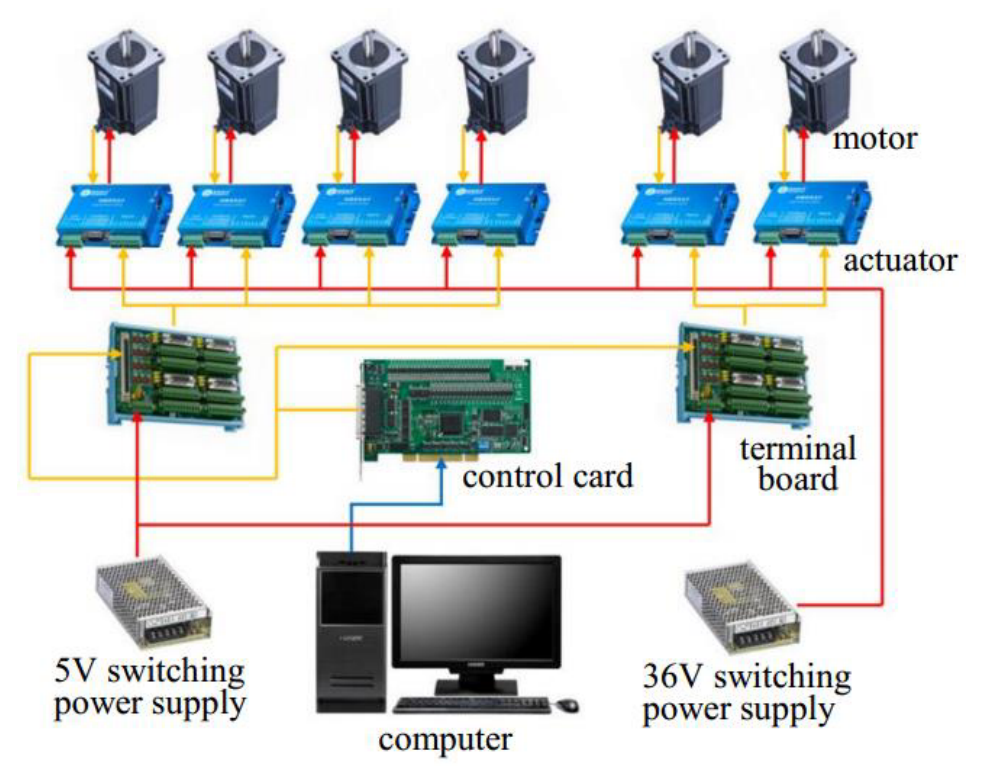

Figure 3. Systematic block diagram of electrical system of the scale crane with the STMAS input signals. The three power wires of the motor are connected to the terminal $\mathrm{U}, \mathrm{V}, \mathrm{W}$ of the actuator whereas its feedback line is connected to 15 -pin DB terminal of the actuator.

\subsection{Design of sway angle measurement device}

In general, sway angle of the hoist line of payload is measured by two encoders ${ }^{[10]}$ which are installed perpendicularly. However, in order to cut down the total expense, the scale crane built in this paper is quite small indeed. The design gross weight of the payload in this paper is only 1 kilogram, so it is not heavy enough to drive the encoders that can be bought when it sways. Another way to measure the sway angle is by several cameras ${ }^{[11]}$ and their image analysis, but it is quite expensive and complicated. In this paper, a sway angle measurement device is designed and built based on Inertial Measurement Unit (IMU). The IMU and its pin definitions are shown in Figure 4 and Table 1.

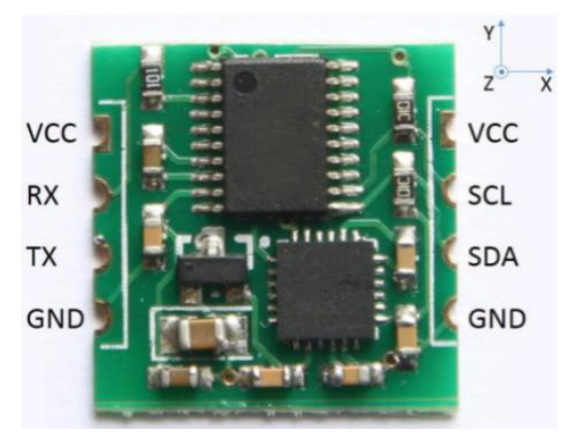

Figure 4. The IMU

Table 1. Pin Definitions of the IMU.

\begin{tabular}{ll}
\hline Pin & Definitions \\
\hline VCC & Power supply, $3.3 \mathrm{~V}$ or $5 \mathrm{~V}$ \\
GND & Ground \\
RX & Serial data in \\
TX & Serial data out \\
SCL & $\mathrm{I}^{2}$ C clock line \\
SDA & $\mathrm{I}^{2} \mathrm{C}$ data line \\
\hline
\end{tabular}

The IMU can be used in three methods:

(1) Use a microcontroller (MCU) to communicate with it by $\mathrm{I}^{2} \mathrm{C}$ bus;

(2) Use a USB serial module to connect it to the computer;

(3) Use its own embedded blue tooth module to communicate with the computer.

In order to prevent the signal lines from dragging the payload, method (3) is selected in this paper. Then the IMU and its battery are installed in an aluminum support. Finally, the communication between the IMU and the computer is debugged. The overall structure of the sway angle measurement device is shown in Figure 5 .

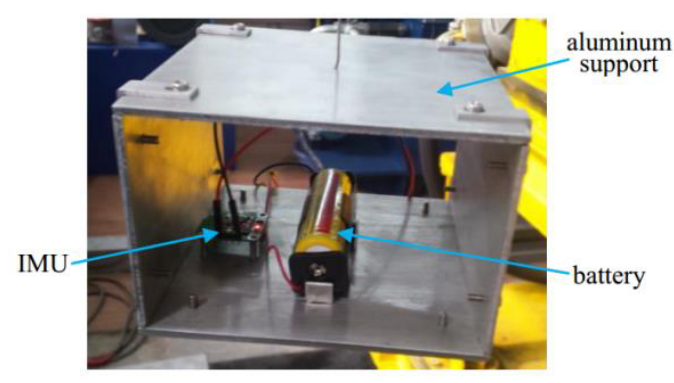

Figure 5. The sway angle measurement device 


\section{MATEC Web of Conferences}

\subsection{Overall Structure of the anti-sway test bed}

On the basis of proposed STMAS, a scale crane is built and tested. Its structure is shown in Figure 6.
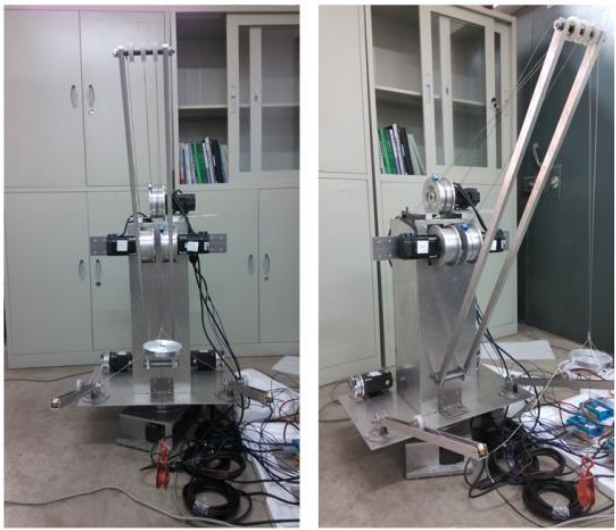

Figure 6. Scale crane with the STMAS

The scale crane shown in Figure 6 is just a land based crane, so the next step to build an offshore crane is to install the scale crane on a ship motion simulator. So an iron support is designed and built considering the real situation of the ship motion simulator. Then the scale crane is installed on the iron support. Finally, the sway angle measurement is hanged by the hoist line as the payload at the same time. The overall structure of the anti-sway test bed for offshore crane is shown in Figure 7.

As shown in Figure 7, the test bed consists of a ship motion simulator, a scale crane, a STMAS and a sway angle measurement device. Among them, the ship motion simulator was built two years ago, whereas others are newly built. The ship motion simulator is driven by four symmetrically arranged cylinders to simulate roll and pitch of the ship, and the hydraulic system above its platform is designed to simulate a hydraulic propulsion system and has nothing to do with this paper. The scale crane is installed on the platform of the ship motion simulator and can carry out slew, luff, and hoist operations like actual crane. The sway angle measurement device can measure the roll and pitch of the payload based on the IMU, and then the measurement results are sent to the computer by blue tooth. In fact, it is obvious that the roll and pitch angle of the payload are equal to its in-plane and out-plane sway angle.

\section{SYNCHRONIZATION BETWEEN THE SLING TRAY AND THE PAYLOAD}

\subsection{Schematic diagram of STMAS}

The schematic diagram of STMAS is shown in Figure 8. Axis $\mathrm{Z}$ represents the axis of crane slew, $\mathrm{AE}$ the guide arm, $\mathrm{BC}$ the boom, $\mathrm{D}$ the sling tray, $\mathrm{P}$ the payload and $\mathrm{C}$ the crane tip. $Z_{\mathrm{PC}}$ represents the radius of the pendulum motion without the STMAS, and $Z_{\mathrm{PD}}$ represents the radius of the pendulum motion with the STMAS. When $Z_{\mathrm{PD}}<Z_{\mathrm{PC}}$, thus the STMAS shortens the radius of the pendulum motion as mentioned above. However, on one hand, if the position of $\mathrm{D}$ is not synchronous with $\mathrm{C}$ when carrying out hoist operations, this will lengthen $Z_{\mathrm{PD}}$, thus reducing the anti-sway performance of the STMAS; on the other hand, if the position of $\mathrm{D}$ is not synchronous with $\mathrm{C}$ when carrying out luff operations, this may loosen or tighten or even break the suspension line and the tagline. Therefore, the position of D must be controlled synchronously with $\mathrm{C}$, and this will be analyzed in the following passages.

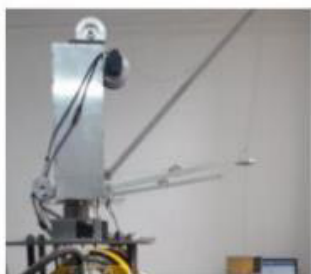

scale crane

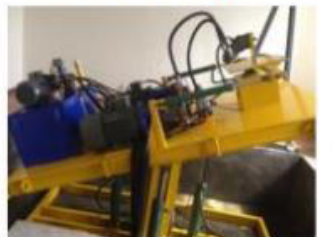

ship motion simulator

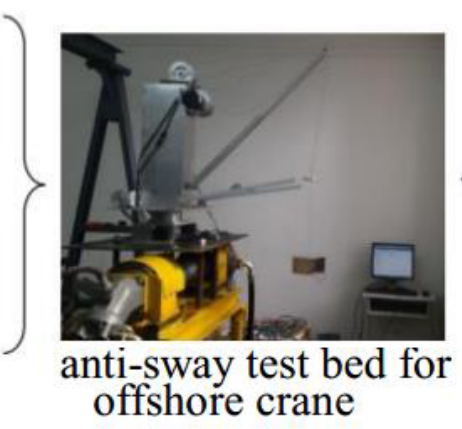

offshore crane

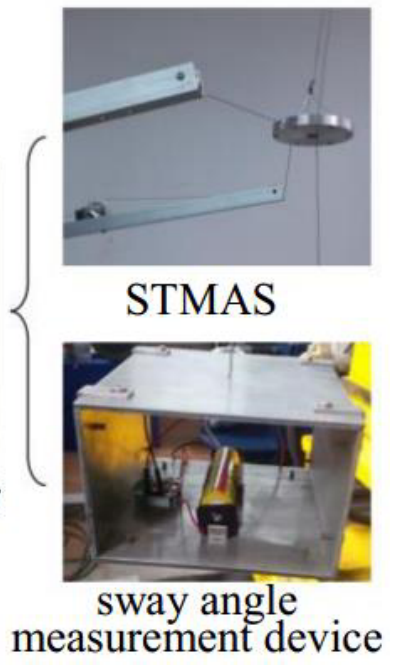

Figure 7. Overall structure of the anti-sway test bed for offshore crane. 


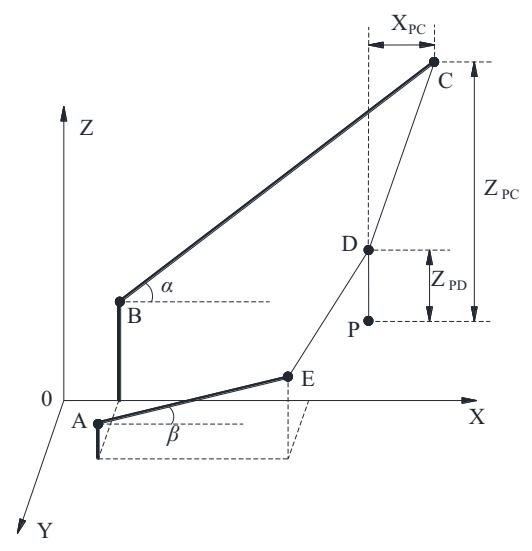

Figure 8. The schematic diagram of the STMAS

\subsection{Mathematic model of synchronization}

The synchronization can be described as: the relative position of the sling tray and the payload must remain unchanged when carrying out luff and hoist operations. This means $Z_{\mathrm{PD}}$ and $X_{\mathrm{PC}}$ are constant, as shown in Figure 8 . The mathematic problem of the synchronization can be concluded as: finding the tagline length $L_{\mathrm{DE}}$ and the suspension length $L_{\mathrm{CD}}$ with $\mathrm{A}\left(X_{\mathrm{A}}, Y_{\mathrm{A}}, Z_{\mathrm{A}}\right)$, $\mathrm{B}\left(X_{\mathrm{B}}, Y_{\mathrm{B}}, Z_{\mathrm{B}}\right)$, and $\mathrm{E}\left(X_{\mathrm{E}}, Y_{\mathrm{E}}, Z_{\mathrm{E}}\right)$ as fixed points, the boom length $L_{\mathrm{BC}}$ and the guide arm length $L_{\mathrm{AE}}$ as known values, $Z_{\mathrm{PD}}$ and $X_{\mathrm{PC}}$ as constant, $\alpha$ and $Z_{\mathrm{PC}}$ as variables.

As seen in Figure 8, we can find $L_{\mathrm{CD}}$ :

$L_{C D}=\sqrt{X_{P C}{ }^{2}+\left(\mathrm{Z}_{P C}-\mathrm{Z}_{P D}\right)^{2}}$

$\mathrm{C}\left(X_{\mathrm{C}}, Y_{\mathrm{C}}, Z_{\mathrm{C}}\right)$

$\left(X_{\mathrm{C}}, Y_{\mathrm{C}}, Z_{\mathrm{C}}\right)=\left(X_{\mathrm{B}}+L_{\mathrm{BC}} \cos \alpha, 0\right.$, $\left.Z_{\mathrm{B}}+L_{\mathrm{BC}} \sin \alpha\right)$

$\mathrm{D}\left(X_{\mathrm{D}}, Y_{\mathrm{D}}, Z_{\mathrm{D}}\right):$

$$
\begin{array}{r}
\left(X_{D}, Y_{D}, Z_{D}\right)=\left(X_{\mathrm{B}}+L_{\mathrm{BC}} \cos \alpha-X_{P C}, 0,\right. \\
\left.Z_{\mathrm{B}}+L_{\mathrm{BC}} \sin \alpha-Z_{P C}+Z_{P D}\right)
\end{array}
$$

$\mathrm{E}\left(X_{\mathrm{E}}, Y_{\mathrm{E}}, Z_{\mathrm{E}}\right):$

$\left(X_{E}, Y_{E}, Z_{E}\right)=\left(X_{A}+L_{A E} \cos \beta, Y_{A}\right.$,

$$
\left.Z_{A}+L_{A E} \sin \beta\right)
$$

From equation (3) and (4), we can find $L_{\mathrm{DE}}$ :

$$
\frac{L_{D E}=\sqrt{\left(X_{\mathrm{B}}+L_{\mathrm{BC}} \cos \alpha-X_{P C}-X_{A}-L_{A E} \cos \beta\right)^{2}+Y_{A}^{2}}}{+\left(Z_{\mathrm{B}}+L_{\mathrm{BC}} \sin \alpha-Z_{P C}+Z_{P D}-Z_{A}-L_{A E} \sin \beta\right)^{2}}
$$

In conclusion, $L_{\mathrm{DE}}$ and $L_{\mathrm{CD}}$ need to satisfy equation (5) and (1) respectively when carrying out luff and hoist operations.

\section{ANTI-SWAY EXPERIMENT}

\subsection{Experimental conditions}

On the basis of the anti-sway test bed for offshore crane built as shown in Figure 7, the experiments are carried out to testify its performance. The experimental conditions are as follows:

(1)The ship motion roll $\varphi_{\mathrm{r}}$ and pitch $\varphi_{\mathrm{p}}$ :

$$
\varphi_{r}=5 \sin \left(\frac{1}{5} \pi \mathrm{t}\right) \quad \varphi_{p}=2 \sin \left(\frac{1}{5} \pi \mathrm{t}\right)
$$

(2)Crane slew operation: velocity $1.8^{\circ} / \mathrm{s}, 0-36^{\circ}$ reciprocating motion;

(3)The total length of the rope is set as $140 \mathrm{~cm}$;

(4)Lift or lower the sling tray to set the radius of pendulum as: $L=140 \mathrm{~cm}, 120 \mathrm{~cm}, 80 \mathrm{~cm}, 40 \mathrm{~cm}$, and define these experiments as E1-E4 respectively.

\subsection{Experiment results}

In Figure 9, if $\mathrm{P}$ sways to $\mathrm{P}$ ', the definitions of out-plane angle $\theta 1$ and in-plane $\theta 2$ are shown in Figure 9. Angles $\theta 1$ and $\theta 2$ denote the payload sway angles in the plane of vertical boom motion and the tangential direction of horizontal boom motion. Angles $\theta 1$ and $\theta 2$ are equal to the roll and pitch angle measured by IMU, the results are shown in Figure 10-11.

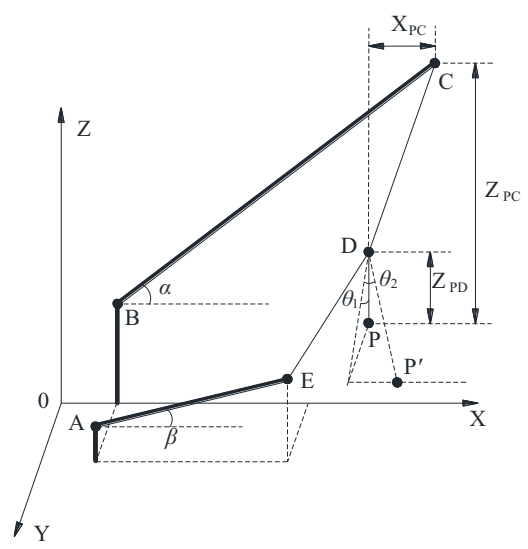

Figure 9. The definitions of out-plane angle $\theta_{1}$ and in-plane $\theta_{2}$.

In Figure 9 , if $\mathrm{D}$ and $\mathrm{P}$ are fixed, the projection of DP' along axis $\mathrm{X}$ is $L \sin \theta_{2}$, the projection of DP' along axis $\mathrm{Y}$ is $L \cos \theta_{2} \sin \theta_{1}$, and then the sway amplitude of $\mathrm{P}^{\prime}$ 'in horizontal direction can be described as $\left(L \sin \theta_{2}, L \cos \theta_{2} \sin \theta_{1}\right)$. Substituting the measurements in E1-E4, the results are shown in Figure 12. 


\section{MATEC Web of Conferences}

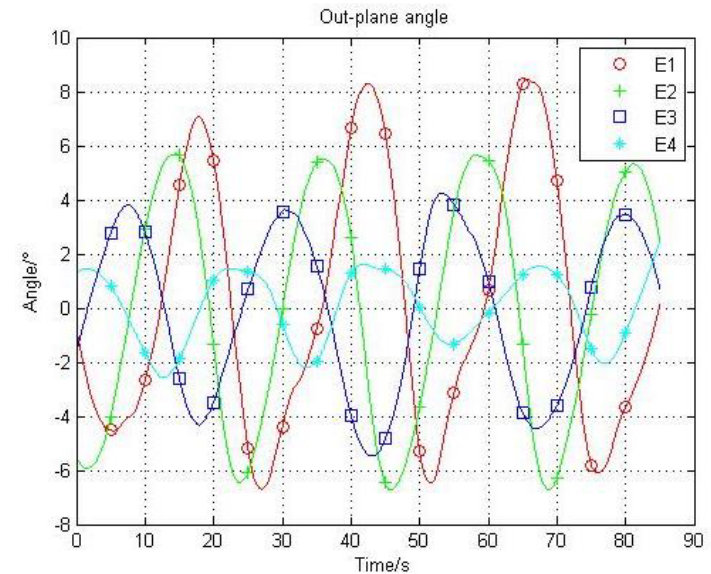

Figure 10. Angle $\theta_{1}$ in E1-E4.

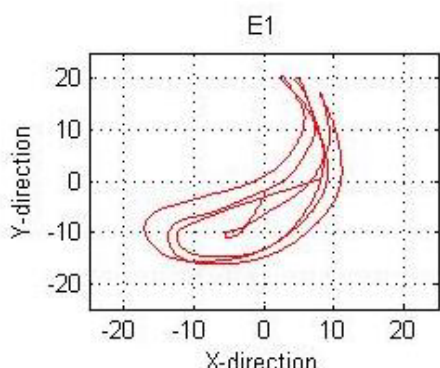

E3

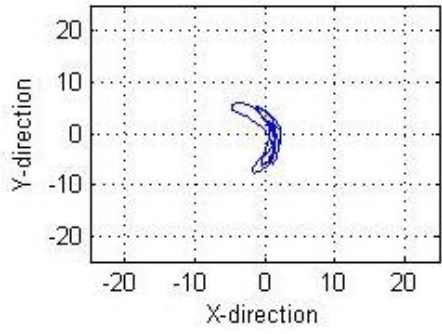

Figure 12 . The sway amplitude of $\mathrm{P}^{\prime}$ in horizontal direction.

\subsection{Analysis of experimental results}

From Figure 10-11, it can be found that:

(1) As the radius of pendulum decreases, the out-plane angle and in-plane angle of payload sway decrease as well, which illustrates that the anti-sway performance improves as the distance between the sling tray and the payload is shortened;

(2) In E1-E4, the out-plane angle are bigger than the in-plane angle, which is because ship roll amplitude is bigger than pitch amplitude, and ship roll is the main

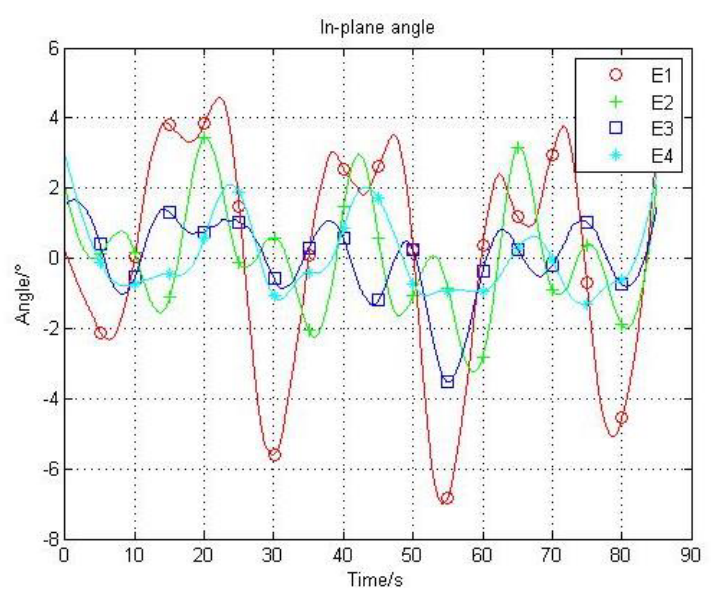

Figure 11. Angle $\theta 2$ in E1-E4.

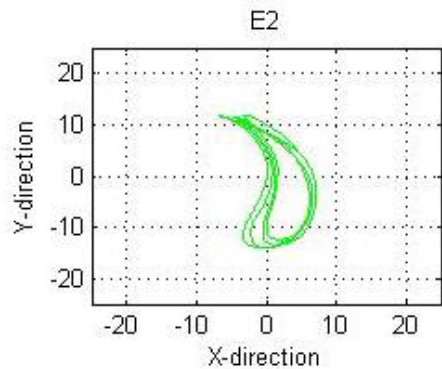

E4

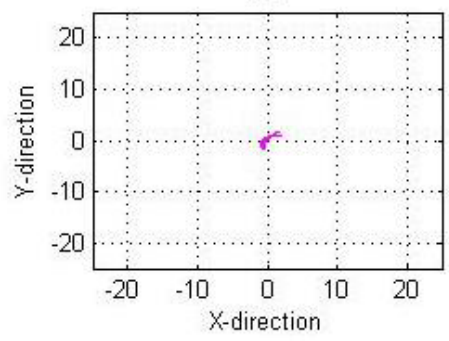

disturbance of out-plane angle while ship pitch is the main disturbance of in-plane angle.

From Figure 12, it can be found that:

(1) As the radius of pendulum decreases, the payload sway amplitudes in horizontal direction decrease significantly, which also illustrates that the anti-sway performance improves as the distance between the sling tray and the payload is shortened;

(2) E1 is equal to the situation without the STMAS, and the sway amplitude can be $20 \mathrm{~cm}$ in $\mathrm{Y}$-direction, but when the radius decreases to $40 \mathrm{~cm}$ in $\mathrm{E} 4$, the sway amplitude can only be $3 \mathrm{~cm}$, which illustrates that the anti-sway performance of the STMAS is obvious. 
ICETA 2015

In fact, Figure 12 is calculated based on the measurements in Figure 10-11, but it demonstrates the anti-sway performance more distinctly.

\section{CONCLUSION}

In this paper, a sling tray mechanical anti-sway system (STMAS) is proposed. On the basis of the proposed STMAS, an anti-sway test bed for offshore crane is designed and built. Then synchronization between the payload and the sling tray is analyzed and its mathematic model is built. Finally, the anti-sway experiments are carried out to testify the performance of the proposed STMAS. Conclusions are drawn from these experiments: the anti-sway performance is obvious after installing the STMAS; in addition, the anti-sway performance improves as the distance between the sling tray and the payload is shortened.

\section{ACKNOWLEDGEMENTS}

This paper is sponsored by the Fundamental Research Funds for the Central Universities (3132015032, 3132015025 ) and the Research Projects of Education Department of Liaoning Province (L2012170).

\section{REFERENCES}

[1] Pengcheng Wang, Yongchun Fang. \& Jilei Xiang, et al. 2011.Dynamics Analysis and Modeling of Ship-mounted Boom Crane. Journal of Mechanical Engineering, 47(20): 34-40.

[2] Kimiaghalam B, Homaifar A. \& Sayrrodsari B.2001. An applicaton of model predictive control for a shipboard crane. Proceedings of the 2001 American Control Conference, Arlington, USA, June: 929-934.

[3] Wen B, Homaifar A. \& Bikdash M, et al.1999. Modeling and optimal control design of shipboard crane. Proceedings of the 1999 American Control Conference, San Diego, USA, June: 593-597.

[4] Parker G, Graziano M. \& Leban F, et al.2007. Reducing Crane Payload Swing Using A Rider Block Tagline Control System. OCEANSE, Aberdeen, June: 1-5.

[5] M. Agostini, G. G. Parker. \& K. Groom, et al.2002. Command shaping and closed-loop interactions for a ship crane. The 2002 American Control Conference, Anchorage, AK, May 8-10: 2298-2304.

[6] Z. N. Masoud, A. H. Nayfeh. \& D. T. Mook.2004. Cargo Pendulation Reduction of Ship-Mounted Cranes. Nonlinear Dynamics, (35): 299-311.

[7] Jang Jae Hoon , Kwon Sung-Ha. \& Jeung Eun Tae. 2012. Pendulation reduction on ship-mounted container crane via T-S fuzzy model. J. Cent. South Univ, (19): 163-167.

[8] R. M. T. Raja Ismail. \& Q. P. HaTrajectory.2013. Tracking and Anti-sway Control of Three-dimensional Offshore Boom Cranes Using Second-order Sliding Modes. IEEE International Conference on Automation
Science and Engineering (CASE), Madison, WI, 17-20 Aug: 996-1001.

[9] Yongchun Fang, Pengcheng Wang. \& Ning Sun, et al. 2014.Dynamics Analysis and Nonlinear Control of an Offshore Boom Crane. IEEE Transactions on Industrial Electronics, 61(1): 414-427.

[10]R.J. Henry, Z.N. Masoud. \& A.H. Nayfeh, et al. 2001 Cargo Pendulation Reduction on Ship-Mounted Cranes Via Boom-Luff Angle. Journal of Vibration and Control, 7: 1253-1264.

[11]Kelvin Chen, Chih Peng. \& William Singhose, et al. 2012. Initial Investigations of Hand-Motion Crane Control with Double-Pendulum Payloads. 2012 American Control Conference, Montréal, Canada, June: 6270-6275. 